\title{
The Current State of Autography
}

\section{Author}

Dr Kostas Grigoriadis

\author{
Affiliation \\ Architectural Association (AA) School of Architecture
}

\section{Address}

36 Bedford Square, London WC1B 3ES

\section{Email}

\section{grigoriadis@aaschool.ac.uk}

\begin{abstract}
As the practice of using notations to translate from two to three-dimensions is gradually being replaced by the direct, autographic relaying of building information digitally, the separation between designing and building is diminishing. Key to lessening further this division are heterogeneous materials, the imminent use of which can instigate the gradual superseding of building components and effectively tectonic construction. Pre-empting their anticipated widespread application, a main point syntax is presented of the expected changes that will occur in architecture as a result. Following this, a novel design method of using particle system elements to simulate the fusion of materials is deployed in the redesign of a building facade element through a multi-material. The ensuing focus is the fabrication of the element, which is performed by converting material data from the CFD program into a 3D-printable format. The current technical limitations of architectural autography are discussed through this workflow.
\end{abstract}

\section{Keywords}

autography; allography; architecture; digital design; multi-materials; functionally graded materials; particle system elements; cfd; rapid manufacturing; $3 \mathrm{~d}$ printing; design theory.

\section{Biographical Note}

Dr Kostas Grigoriadis studied Architecture at UCL, followed by a Master in Architecture and Urbanism at the Architectural Association's Design Research Laboratory. He has been a Diploma Unit Master at the AA since 2011 and an External Examiner in Architecture at the University of East London since August 2015. He previously worked for Foster and Partners in London and held a Visiting Lectureship at the Royal College of Art where he also completed a PhD in Architecture by Project in June 2017 that focused on multi-material design methodologies. He has edited the book 'Mixed Matters: A Multi-Material Design Compendium', published in June 2016 by Jovis Verlag.

\section{Introduction}

\subsection{Allography \& Autography}

When discussing the separation in architecture between designing and making, Tim Ingold (2013, p.49) notes that this was instigated back in the fifteenth century by Alberti, who stood 
as "a pivotal juncture in the process that ultimately led to the professionalisation of architecture, as a discipline devoted exclusively to design as opposed to implementation."

This division stemmed from the hylomorphically biased definition of the architect as a "man of 'learned intellect and imagination', who is able 'to project whole forms in mind without any recourse to the material'" (Ingold, 2013, p.49). In this one-directional allographic (Carpo, 2011) process, mental forms stemming in the designer's intellect are transposed upon the material world through a series of notational and numerical instructions or 'lineaments', eventually giving rise to physical constructs through the mediation of workmen.

Moving this forward to a contemporary context, "Alberti's authorial and notational method of design has become a staple of modern life" (Carpo, 2011, p.77), as mass production, material homogenisation, outmoded construction methods and what can be termed as the flat-sheetorigin of the majority of building components, dictate the need for allographic translation between two and three dimensions. This is because buildings developed as holistic forms in the design process must be discretised into innumerable flat sheet elements, that are bent, folded and mechanically assembled back together "in a process of chaos and conflict" (Wiscombe, 2012, p.1) through immensely time-, cost-, and energy-consuming processes, in order to give rise again to the totality of the building.

\subsection{Large Scale 3D Printing \& Material Fusion}

At the same time, there are two main technical developments that are beginning to fundamentally question both the practice of notation and discretisation, as well as the hylomorphic, materially-disengaged design process. The first one is regarding advanced forms of materiality that supersede the need for mechanical assemblage through material fusion, and the second concerns novel construction techniques that can enable building parts or even whole buildings to be rapidly manufactured.

Regarding the former, the practice of fusing materials together on visible scales was instigated as far back as the nineteen seventies in aeronautic and material science research. The problem of very high stresses building up in the connections between the metallic and ceramic parts deployed in hypersonic space planes, was resolved by developing a new type of (functionally graded (FGM) or multi-) material, consisting of ceramic fusing into steel continuously over its volume. That way the thermal insulation properties of ceramics were combined with the structural properties of metals, eschewing the use of any mechanical fasteners or joining that would compromise the integrity of the material system.

Fast forwarding to a contemporary context, recent research initiatives similarly indicate that the transferring of material gradation from fields such as aerospace engineering to the construction industry can enable savings both in terms of energy, as well as in material quantities, while eliminating the formation of weak points in the places that parts would connect in a conventional manner (Oxman, Keating and Tsai, 2011; Federal Ministry of Transport, Building and Urban Development, 2010). Effectively enabling the superseding of tectonic 'collaging', this transfer has been heralded as "a sea change in the world of construction: [a] shift from assemblage to fusion [emphasis added]. In material terms this translates into a move from mechanical to chemical attachments; more simply, things are built without bolts, screws, nails, and pegs"1 (Lynn and Gage, 2010, p.20).

\footnotetext{
${ }^{1}$ Lynn was referring at the time to composite materials, however, the quote is still valid in the present discussion about functionally graded materials.
} 
Regarding the aforementioned construction technique novelty, research programmes like the LASIMM (Large Additive Subtractive Integrated Modular Machine) project, which is a collaboration between Foster and Partners, the European Welding Federation and BAE Systems among others, funded through the EU Horizon 2020 initiative, are investigating the potential widespread application of 3D printing in the building industry. Working towards a future in which "entire buildings will be 3D printed" (Dunton, 2017), this research is also strong evidence of a turn from the aforementioned flat-sheeting practice towards integrated, direct and autographic (Carpo, 2011) construction. This is additionally because "contemporary CAD-CAM technologies have obliterated the notational gap that for centuries kept design and construction apart"2 (Carpo, 2011, p.78), allowing for a (theoretically) seamless workflow between design and construction.

\subsection{The Eight Point Syntax of Architectural Multi-Materiality}

With all this in mind, and in the context of a gradual shift towards the incorporation and application of fused materiality in architecture and design, it is logically envisaged that the following will be the fundamental changes occurring as a result:

a. Tectonic construction, based on the assemblage of materially uniform, discrete building components, will progressively be superseded.

b. Fusing will become the appropriate building technique linked to this twenty first century (multi-)material paradigm.

c. Discrete boundaries will be replaced by gradients.

d. The acceptable margin for error will increase, as gradients are by definition more 'forgiving' than discrete components.

e. Designing and building will effectively be made with multi-materials directly.

f. There will be a shift towards a new design process in which material behaviour will be prioritized.

g. Procedures of allographic translation between two and three dimensions, in which 3D CAD information is typically converted into 2D (drawn) instructions that then get converted again into built 3D space, will be superseded by an autographic and seamless process of designing and making.

h. Principles of composition previously based on the arrangement of discrete geometric elements will effectively have to be rethought.

\section{Designing \& Fabricating Fusion}

\subsection{Research Subject Definition}

Correspondingly, with the process of designing and 3D printing a materially-uniform object being nowadays a straight-forward endeavour, the discussion that is going to follow will shift towards multi-material fabrication and will mainly be concerning point $g$. It will effectively be a technical investigation into the current state of architectural autography, namely highlighting the three main procedural problems encountered in converting a digitally and multi-materially designed building element directly into a materially-graded physical artefact. The intent will be to initiate a focused discussion on the technical problems that need to be overcome, capturing a snapshot in the trajectory towards design and making convergence, and towards building component fusion.

\footnotetext{
${ }^{2}$ This quote is referring to direct data relaying from the computer to the fabrication facility, but at the same time still regarding discrete (albeit three dimensional) components as outputs.
} 
Before proceeding to the digital to physical conversion workflow, it should be mentioned that the initial design operation is concentrated on a building envelope detail, namely the connection of glazing to its surrounding aluminium frame in a standard, widely used off-theshelf cladding panel (in this case model FW 50+SG from the Schüco Facade System catalogue). The connection is essentially a standard mechanical assembly of a multitude of discrete homogenous materials that is correspondingly redesigned through a single multimaterial that preserves the structural and optical qualities of the original panel. Briefly considering the above-mentioned points $e$. and $f$., the part that follows will touch upon the software utilised, sub-materials used, and design principles followed in the redesign of the connection.

\subsection{Appropriate CAD Software Identification}

When investigating commercially available techniques (eschewing a long-drawn discussion on custom software creation), research has shown that voxels and particle system elements (Oxman, 2011; Knoppers et al., 2005) are the two main options for incorporating multimaterial information in the computer. A critique of the former, however, is that it does not take into account material properties in the software environment, but rather assigns colour data as representational place holders for materials. Particle systems on the contrary, are by virtue of their computational structure made to simulate physical phenomena and effectively material behaviour, and have therefore been utilised to simulate sub-material fusion in a functionally graded material.

\subsection{Sub-Material Selection}

In terms of appropriate substances to be used in the graded mix, the discrete parts that typically make up the aforementioned cladding panel consist of aluminium, silicone, polyetherimide, EPDM (ethylene propylene diene polymethylene) rubber and insulating glass. When designing with a multi-material, a main consideration is the fusion compatibility between its constituent sub-materials. In this regard, initial research showed that there are no present-day cases in which glass has been made to fuse with aluminium in an FGM directly. Further investigation, however, indicated that it is possible to fuse together aluminium with alumina (Birman and Byrd, 2007), namely in "aluminum matrix composites reinforced by nanoceramic particles" (Mahboob, Sajjadi and Zebarjad, 2008, p.240). In addition, there currently exists "a method of percolating [...] molten $\mathrm{CaO}-\mathrm{ZrO}_{2}-\mathrm{SiO}_{2}$ glass into [...] [a] polycrystalline sintered alumina substrate to prepare glass-alumina functionally graded materials" (Yu, et al., 2007, p.134). With this in mind, it would be theoretically, as well as technically feasible to deploy alumina as an interface material subset in an aluminiumalumina-glass functionally graded material entity.

\subsection{Fusion Simulation}

Consequently, the dynamics simulation platform RealFlow by Next Limit Technologies, which consists of "a complete set of simulation engines that cooperate to solve complex scenes with multiple interactions among elements of different nature" (Vučković, 2009) was used for the simulation. Each material was assigned a corresponding particle system comprising of values such as density, viscosity and internal pressure that can all be attributed to accurately simulate physical material behaviour. In addition, as fusion occurs with liquid substances, a b-rep element was designed and imported into the software in order to contain the materials to be merged (or the fluids would keep falling in infinite virtual space under the influence of gravity). 


\subsection{A New Design Process}

Going back to the initial discussion about one-directional form imposition on a material substrate, the main departure from the standard design process here, is that materiality is incorporated directly at an early stage and allowed to contribute with its own agency to gradient formation. Gradients are effectively allowed to self-formulate within the virtual bounds delimited by the rigid framework of the designed container and the rheological behaviour of the simulated materials. The combination of container design and material flow in this instance is a loose type of control or something akin to "controlling, but resisting control" (Wiscombe, 2010, p.21). In effect, the focus shifts from the design practice of "drawing as an assembly of 'lineaments' [emphasis added]" (Ingold, 2013, p.125) towards the one of computational material "cooking in a bag [emphasis added]" (Lynn and Gage, 2010, p.19).

\subsection{Simulation Set-up}

In terms of the particularities of the procedure, six particle emitters in total (two each for aluminium, alumina and glass) were placed inside the containing geometry and the corresponding values attributed accordingly: an approximate density of $2,500 \mathrm{~kg} / \mathrm{m}^{3}$ and dynamic viscosity of $100 \mathrm{~Pa} \cdot \mathrm{s}$ for melted glass, density of $2,830 \mathrm{~kg} / \mathrm{m}^{3}$ and dynamic viscosity of $0.0054 \mathrm{~Pa} \cdot \mathrm{s}$ for alumina, and $2,375 \mathrm{~kg} / \mathrm{m}^{3}$ and $0.001379 \mathrm{~Pa} \cdot \mathrm{s}$ for aluminium.

The simulation was then initiated with alumina placed in the central compartments and aluminium and glass on either ends of the container. The blending reached the required formation $^{3}$ after approximately ten seconds and eventually terminated at twelve.

\subsection{The Current State of Autography}

\subsubsection{Colour Representation of Materials}

The resulting blend was made up of a point cloud that had to be converted into a CAD geometry for it to be eventually fabricated. This was possible as RealFlow has an in-built way of converting particle systems into b-rep geometries (meshes) that can be output in other programs, typically for visualisation purposes (bearing in mind that the software is primarily used in the advertising and visual effects industries). This in turn means that one must revert to colour representation as opposed to working with the original material attributes. This is because a mesh only allows for colouration as the main way of distributing and visualising gradients over its otherwise material-less topology. The difference to the above-mentioned voxel approach, however, is that gradient formation is based on emulating physical material flow, with gradients only represented in the form of colours after the simulation is terminated and the final arrangement frozen in time.

A unified mesh was consequently applied on the simulated particle blend, forming a "threedimensional representation of the outmost particles of [...] [the] emitters" (RealFlow 2014 Documentation: Nodes - Meshes). Multi-materiality was imprinted on this mesh skin as colour graded regions in-between the main sub-material areas (represented by different colours) of the mesh (Figure 01).

\footnotetext{
${ }^{3}$ The required formation here referring to the interspersed patterning of the neighbouring materials in the fused regions, which is visually verified and follows the micro-characteristics of a graded structure as described in extensive detail by Miyamoto et al. (1999, p.41).
} 


\subsubsection{Problem 01- Cross Platform Material Data Transfer}

Going back to point $g$. and to the digital to physical conversion workflow, the main problem here was related to the wider phenomenon of CAD program proliferation and differing computational structures that prohibit seamless cross-platform information transferring. The particle mesh had to be exported to another software for multi-material fabrication, but although colouration that represented graded materiality was visible in the simulation software, exporting it to another program was not possible. This was because none of the file formats that could be output from RealFlow preserved the material/colour data. To generate the necessary bridging between simulation and fabrication, the gradients had to be converted into numerical values that could then be imported into a commercial 3D modelling application in order to prepare the file for 3D printing. A point to be made here, is that this numerical conversion that had to take place within the computer was merely due to the problem of software incompatibility, and was of course different to the above-mentioned physical outputting of sets of instructions or projected geometries.

\subsubsection{Fluid Weight Data}

The first attempt towards this conversion was to utilise the per-vertex weight of each particle emitter that could be retrieved as a decimal. The value denotes the degree of influence that the individual particle emitters have on the colouration of each of the vertices of the mesh. The Python script utilised to perform this operation was the following:

for $i$ in range ( 0 , total number of mesh vertices): vertexWeight $=$ ParticleMesh.getFluidsWeightAtVertex(i) file.write $(\operatorname{str}($ vertex Weight) $)$

A first run of the script resulted in outputting all weight data in this format:

[('01_Aluminium Fill_Object', 0.0), ('02_Alumina_Fill_Object', 0.0), ('03_Glass Fill_Object', 0.0), ('04 Aluminium Fill_Object', 0.0), ('05_Alumina_Fill_Object', 0.0), ('06_Glass_Fill_Object', 1.0)], which in this case indicated that the specific vertex colouration would be $100 \%$ influenced by particle emitter 06 .

At another index of the data list corresponding to a different mesh vertex, the output would be: [('01_Aluminium_Fill_Object', 0.0), ('02_Alumina_Fill_Object', 0.0), ('03 Glass Fill Object', 0.0), ('04 Aluminium Fill Object', 0.27106717228889465), ('05 Alumina Fill Object',0.20003880560398102), ('06 Glass Fill Object', $0.52889406681060791)]$, indicating that the specific vertex is influenced by three particle systems with the addition of the numbers amounting to 1 .

When data values were then output and saved in a .doc file, a striking fact would be the very large volume of the file size, which for a total of 297,651 mesh vertices would be 68.1 MB, with the overall word count being 3,411,613 words over 17,576 pages. This would have an impact on file handling times and processing power needed to manage and retrieve the data. Removing the preceding ' $\mathrm{x} \_\mathrm{x}$ _Fill_Object' text before each of the numerical values reduced the file size down to $14.2 \mathrm{MB}, 1,785,906$ words and 6,581 pages.

A sequential correspondence between the fluid data and the mesh vertices meant that in principle, a direct colouration of the imported mesh vertices would be possible. The main problem, however, was that when the data was converted into RGB values although being accurate in terms of the distribution of gradients, the colours themselves were different to the ones of the particle mesh. This was because the weight data was an indication of the 
influence of each sub-colour in the overall pigmentation of the vertex rather than a direct colour value. Colouration would be quite straight forward in the case of a 0.0, 0.0, 0.0, 0.0, $0.0,1.0$ output, as the only influence would be of particle system 06 with an RGB value of $(178,216,239)$. The complications would arise when the aforementioned 0.0, 0.0, 0.0, $0.27106717228889465,0.20003880560398102,0.52889406681060791$ output would correspond to the influence of particle systems 04, 05 and 06, each with a different RGB colour of $(87,87,87),(133,13,83)$ and $(178,216,239)$ respectively (Figure 02$)$.

The initial response to this was to convert each RGB value to an integer and multiply that by the weight data value. RGB $(87,87,87)$ for instance would become 5723991 and then $5723991 * 0.27106717228889465 \approx 154547757$. The idea would then be to perform this operation for the other two remaining particle systems and eventually add together and average out the resulting figures in order to acquire the appropriate colour value. This was of course not possible and in retrospect taking into account that an RGB integer stems from this

formula: RGB Integer $=$ Red $+($ Green*256) $+($ Blue*256*256) an alternative approach would be to multiply each RGB value by the fluid data value: $87 * 0.27106717228889465=$ 23.58284398913383 and convert the results to an integer by applying the results to the formula above: $\mathrm{RGB}$ value $=23.58284398913383+(23.58284398913383 * 256)+$ $(23.58284398913383 * 256 * 256)$. The problem, however, would still lie in the averaging out of the numbers of the other two particle systems in order to obtain the correct colour.

\subsubsection{RGB Values}

Eventually, the complexity of this routine was abandoned in favour of a Python script that would output RGB values from the simulation software directly, developed in collaboration with Next Limit Technologies. With a much more manageable file size of $10.3 \mathrm{MB}$, the output text file would contain three values for $R, G$ and $B$, which would be converted from arithmetic into Digital 8-bit per channel values using the following formula: RGB (Vertex R Value $(0$ to 1$) * 255$, Vertex G Value $(0$ to 1$) * 255$, Vertex B Value $(0$ to 1$) * 255)$ (Figure $03)$.

A last hurdle encountered in this routine was that exporting the mesh from the simulation software as an OBJ file and then importing it in Rhino, would result in the number of the mesh vertices being between three to ten less than the vertices of the original mesh. This in turn meant that the total number of vertices would not correspond to the total number of data values, with this non-correlation leading to incorrect colouration. Consultation with programmers at Next Limit technologies indicated that there was no apparent solution to this problem. After repeated trials and errors, however, the solution was to export using the LWO format, with the total vertex count effectively being matched in both software environments (Figure 04).

Having attained the direct transferring of data from one software into another, the accuracy of the gradient effect was tested out by generating a multi-colour sandstone 3D print, coloured according to the RGB data list. The distribution and colouring of gradients were visually identical to the ones generated in the simulation (Figure 05), however, the objective as mentioned earlier would be to achieve direct multi-material correspondence as opposed to one that is representational.

\subsubsection{Problem 02- Material Gradient Discretisation}

When attempting to translate the computer-generated material gradients into ones that are physical, another main problem to consider is that the $3 \mathrm{D}$ printing of gradients is not yet 
possible. The relevant fabrication methods work with singular sub-materials that are combined to form a larger multi-material entity. The continuously coloured mesh described above would therefore have to be discretised by converting the initially continuous, into stepwise material structures. This conversion would affect some of the attributes of the part, while leaving others intact.

Change of properties would naturally be the case when designing with sub-materials that are computationally different to the ones physically fabricated. At the same time shifting to stepwise distribution would have an effect on the structural behaviour within the multimaterial itself, as there would be the problem of additional forces developing in the submaterial segment boundaries. Visually and aesthetically speaking on the other hand, there would be no evident difference between the continuous and the stepwise. This is because the material selection from the glazed area towards the alumina and aluminium part could be made optically incremental, therefore still maintaining the effect of transitioning gradually from opacity to transparency. Lastly, regarding the structural characteristics of each submaterial, further research is necessary in order to evaluate the pros and cons of replacing glass with transparent plastic and of aluminium with a Polyjet rigid plastic material.

Consequently, this additional operation of converting the continuous to the step-wise was performed by running a VB script on the graded colour mesh and isolating similarly coloured mesh faces. A fact about mesh colouration, however, is that it occurs on face vertices and not on the mesh faces themselves. The RGB colour of each of the three vertices corresponding to a mesh face therefore had to be retrieved, indexed in an array and stored together with the face itself. The stored per-face vertex values would then be averaged out and tested against an input colour value and if the two would equate, all faces within the same colour range removed from the overall mesh topology and placed on a separate layer (Figure 06). Performing the routine with a different input colour value each time, eventually isolated all similar-colour families into different layers (Figure 07).

An additional observation at this point would be that the fineness of each of the colour layers would dependent on two parameters. The first was the size of the polygons that the overall mesh would be made of and the second, the degree of deviation of the average mesh face colour to the input colour value. Smaller polygon sizes resulting in a larger number of mesh faces, as well as a smaller deviation of face to input colour value would mean that the overall mesh would be cut down in very fine, almost imperceptibly small segments that would give a better impression of gradation. The flipside to this would be more sub-material boundaries within the larger mesh topology affecting structural behaviour, as well as the available Polyjet materials not being enough to correspond to the large number of sub-materials. Eventually, the script was run for a total number of nine Polyjet materials (three base materials and six sub-materials) that were corresponding to the original gradients as closely as possible, given the multi-material 3D printing sub-material availability, mesh subdivision limitations and target colour range.

\subsubsection{Achieving Gradients through Discretisation}

In retrospect, as the above-mentioned algorithm was working with median values, it became apparent that the output obtained from the fluid weight data routine would have sufficed for converting to a step-wise distribution. This is because precise colouration was not critical, since accurate relationality between the types of gradients and colours would have been enough for discretising the mesh in a successful manner. 
Furthermore, regarding the gradient effect attained, bearing in mind the objective of maintaining the appearance of gradients despite the discretisation that had to occur, the face colour averaging routine came with its own inconsistencies. The original gradients were formed namely between the aluminium and alumina (grey and red) and alumina and glass (red and light blue) regions. The inconsistency in this instance occurred when a mesh face in the aluminium and alumina fusion region would exhibit the same median RGB value, as a face in the alumina and glass area. The algorithm would consider these of the same colour family and therefore a continuous mesh segment would be extracted from the overall mesh. Applying a sub-material that would enable gradual sub-material transition between the clear (glass) and alumina areas, however, would not necessarily enable the same visual transition in the aluminium to alumina region.

A simple solution to this would be to initially split the continuous mesh segment into discrete clusters and perform a visual correspondence between the original continuous gradation and its discretised equivalent. The discrete clusters corresponding to the original "pools" of aluminium dispersed within the alumina matrix would be isolated and a new set of submaterials applied to these material pools. As per the PolyJet multi-material printing capabilities, the maximum number of base materials that can be simultaneously 3D printed are three. The ensuing range of "material properties ... from rigid to flexible and clear to opaque in a wide range of colours and hues" (PolyJet Multi-Material 3D Printing) is within the domain defined by these three base material extremes. Apart from colour gradation the other main quality to be attained was transparency gradation, therefore the cyan-magentatransparent palette was selected from the four colour-transparency rigid PolyJet palettes available.

With most of the available sub-materials (six out of seven in total) between the VeroMagenta and VeroClear base materials having already been assigned to the corresponding alumina and glass fusion region, there had to be an assigning of different sub-materials to achieve the appropriate effect in the aluminium and alumina graded boundary region. In that area, there were four sub-material segments in-between VeroCyan (aluminium) and VeroMagenta (alumina), with each one having to in turn correspond to one of seven available sub-materials between these two base materials in the colour chart.

Having corresponded and replaced these accordingly, the resulting distribution exhibited a progressive gradation from VeroCyan (aluminium) to VeroMagenta (alumina) to VeroClear (glass) (Figure 08).

\subsubsection{Problem 03- Mesh Thickening}

Having followed the above workflow, the mesh discretised and the corresponding materials applied to each segment, a further problem was that the depthless mesh shells had to have a certain thickness for 3D printing. Offsetting these to the $0.4 \mathrm{~mm}$ minimum acceptable depth, meant that some of the neighbouring segments would intersect with one another and the corresponding sub-materials would cancel each other out (resulting in the printing of only the three base materials) (Figure 09). This was because following segmentation of the mesh, the edge vertices of each part would exhibit different normal orientations to the end vertices of their adjacent strips.

The workaround to this problem was devised in collaboration with Marios Tsiliakos (from Digital [Sub]stance and the University of Innsbruck), who generated a definition in Grasshopper 3D for offsetting each segment in a corresponding manner. According to Tsiliakos, the workflow consisted of a process of joining the discrete parts back into a single 
mesh, welding all vertices, cleaning the mesh to attain a comprehensive topology, and applying the colouration of the initial sub-material parts to the joined mesh. The normals of the mesh were then unified by average approximation (of neighbouring vertices) and the mesh was offset by a user specified distance (the above mentioned $0.4 \mathrm{~mm}$ being the minimum). The relevant colours were also applied to the offset mesh using a face-bymajority-rule between the corresponding vertices of both the original and offset meshes. Following the extraction of any remaining naked edges, the grouping of them in pairs and the creation of quad mesh faces in-between these edges to generate the perimeters, all original discrete colour, offset and perimeter meshes were joined into watertight mesh clusters. These were assessed for colour inconsistencies (if any were present, the prevailing colour would be applied to the whole cluster), grouped by colour, and each one assigned to a new layer with the colours applied as meta-data ready for 3D printing (the diagram of the full procedure can be found in Figure 10).

Initial testing indicated that these thickened mesh segments can result in a workable multimaterial print. Currently at the stage of manually correcting any remaining intersecting regions, the imminent final step will be to fabricate the part and visually inspect for resemblance or deviations from the digital artefact.

\section{Conclusion \& Discussion}

With the demise of 2D not being "far off" and the use of "on-site robotics and large-scale 3D printing" (Sinclair, 2017, p.76) expected to instigate fundamental changes to the design and build process, multi-materiality can be said to offer further radical possibilities of new design approaches, direct building-data streaming, and building component fusion.

Currently, however, "there is nothing out there that can do true multi-material manufacturing" (Zolfagharifard, 2013). At the same time, it is envisaged that "real innovations will happen in the research world. Multi-material is the next evolution in the technology... you're probably looking at a 5-10 year timescale to see real multi-material integration" (ibid., 2013). As developments in the relevant manufacturing techniques are rapidly advancing towards this integration, architectural autography is expected to become increasingly apropos as a research subject.

Anticipating this imminent reality, a breakdown of the expected changes that will occur in architecture as a result was presented. This was followed by an alternative FGM design method of simulating the fusion of liquid materials using particle system elements. The ensuing computational workflow was an attempt towards the above-mentioned abolition of 2D representation and towards a direct design-to-fabrication procedure.

As part of this process, intermixed, geometry-less material particles had to be converted into a colour graded mesh, which due to cross-platform incompatibilities was exported as a colour- and graded-less b-rep element. Correspondingly, the colour gradients had to be converted into RGB numbers, so that they could be repainted back on the mesh topology. Due to gradients not being 3D printable, the b-rep was then discretised into several singlecolour and single-skin individual meshes, that had to be joined together again, offset and discretised yet again into several single-colour b-rep chunks in order to be finally output ready for multi-material fabrication.

The resulting functionally graded artefact consisted of representational materials that had different properties to the ones originally computed. In retrospect, a potential workaround to this material representation could have been to simulate material fusion using particles with 
the liquid properties of the output Polyjet plastics as opposed to aluminium, glass, and alumina. As these plastics on the other hand, have questionable structural properties when it comes to build application, standard FGM manufacturing methods such as centrifugal casting could have been utilised instead of 3D printing. As these methods only take place at industrial scales, however, and as the main point has been to investigate the unmediated convergence of design and making, this method would have still meant a separation between the two.

In effect, being in its nascent stages, the process of achieving complete autography is far from straight forward and as it has been evident a large degree of mediation is still the case. Having said that, it is a matter of time until direct autography takes over, but until then it is to be hoped that research as the one presented will add to the theoretical and methodological discussion in the trajectory towards design and building fusion.

\section{References}

Birman, V. and Byrd, L.W. (2007) 'Modelling and analysis of functionally graded materials and structures', Applied Mechanics Reviews, 60(5), pp. 195-216

Carpo, M. (2011) The Alphabet and the Algorithm, MIT Press, Cambridge, Mass.; London.

Dunton, J. (2017) 'Fosters: 'entire buildings will be 3D-printed in the future', Building Design, 07 April 2017 [online] http://www.bdonline.co.uk/news/fosters-\%E2\%80\%98entirebuildings-will-be-3d-printed-in-the-future\%E2\%80\%99/5087079.article (Accessed 15 April 2017)

Federal Ministry of Transport, Building and Urban Development (2010). Building the future: The magazine of the Zukunft Bau research initiative [online]. Federal Ministry of Transport, Building and Urban Development, Berlin. http://intaaivn.org/images/cc/Habitat/background\%20documents/CCHabitat_Building the_Future_Ger many.pdf (Accessed 20 April 2017)

Ingold, T. (2013) Making: Anthropology, Archaeology, Art and Architecture, Routledge, London.

Knoppers, G.E. et al (2005). 'The reality of functionally graded material products' in Intelligent Production Machines and Systems: First I*PROMS Virtual Conference, IPROMS 2005, Amsterdam, Holland, pp. 467-474.

Lynn, G. and Gage, M.F. (Eds.), (2010) Composites, Surfaces, and Software: High Performance Architecture, W.W. Norton, New York; London.

Mahboob, H., Sajjadi, S.A. and Zebarjad, S.M. (2008) 'Synthesis of Al- $\mathrm{Al}_{2} \mathrm{O}_{3}$ Nanocomposite by mechanical alloying and evaluation of the effect of ball milling time on the microstructure and mechanical properties' in ICMN08 2008: International Conference on MEMS \& Nanotechnology 2008 (ICMN08), The Faculty of Engineering, International Islamic University Malaysia (IIUM), Kuala Lumpur, Malaysia, pp. 240-245

Oxman, N., Keating, S. and Tsai, E. (2011), 'Functionally graded rapid prototyping' in Innovative Developments in Virtual and Physical Prototyping: Proceedings of the $5^{\text {th }}$ International Conference on Advanced Research and Rapid Prototyping, Centre for Rapid and Sustainable Product Development of the Polytechnic Institute of Leiria, Portugal, pp. 483-489 
Oxman, N. (2011) 'Variable property rapid prototyping', Virtual and Physical Prototyping, Vol. 6 No. 1, pp. 3-31

RealFlow 2014 Documentation: Nodes - Meshes. [online] http://support.nextlimit.com/display/rf2014docs/Nodes+-+Meshes (Accessed 22 April 2017).

Sinclair, D. (2017) 'Digital: it's not optional', RIBA Journal, April 2017, p.76

PolyJet Multi-Material 3D Printing. [online]

http://usglobalimages.stratasys.com/Main/Files/Technical\%20Application\%20Guides_TAG/ TAG_PJ_MultiMaterial_EN_1015.pdf?v=635969150902140765 (Accessed 04 September 2017).

Vučković, V. (2009) 'Virtual models of Tesla's patents', in $17^{\text {th }}$ Telecommunications Forum (TELFOR 2009), TELFOR, Belgrade, Serbia, pp. 1335-1338

Wiscombe, T. (2012) 'Beyond assemblies: system convergence and multi-materiality', Bioinspiration \& Biomimetics, Vol. 7 No. 1, pp. 1-7

$\mathrm{Yu}$, C.L. et al (2007) 'Integrated liquid-phase sintering of glass-alumina functionally graded materials', Science of Sintering, Volume 39 (Issue 2), pp. 133-144

Zolfagharifard, E. (2013) 'The rise of multi-material 3D printing' [online] http://www.theengineer.co.uk/channels/design-engineering/in-depth/the-rise-of-multimaterial-3d-printing/1016242.article (Accessed 30 October 2016)

\section{Figures}




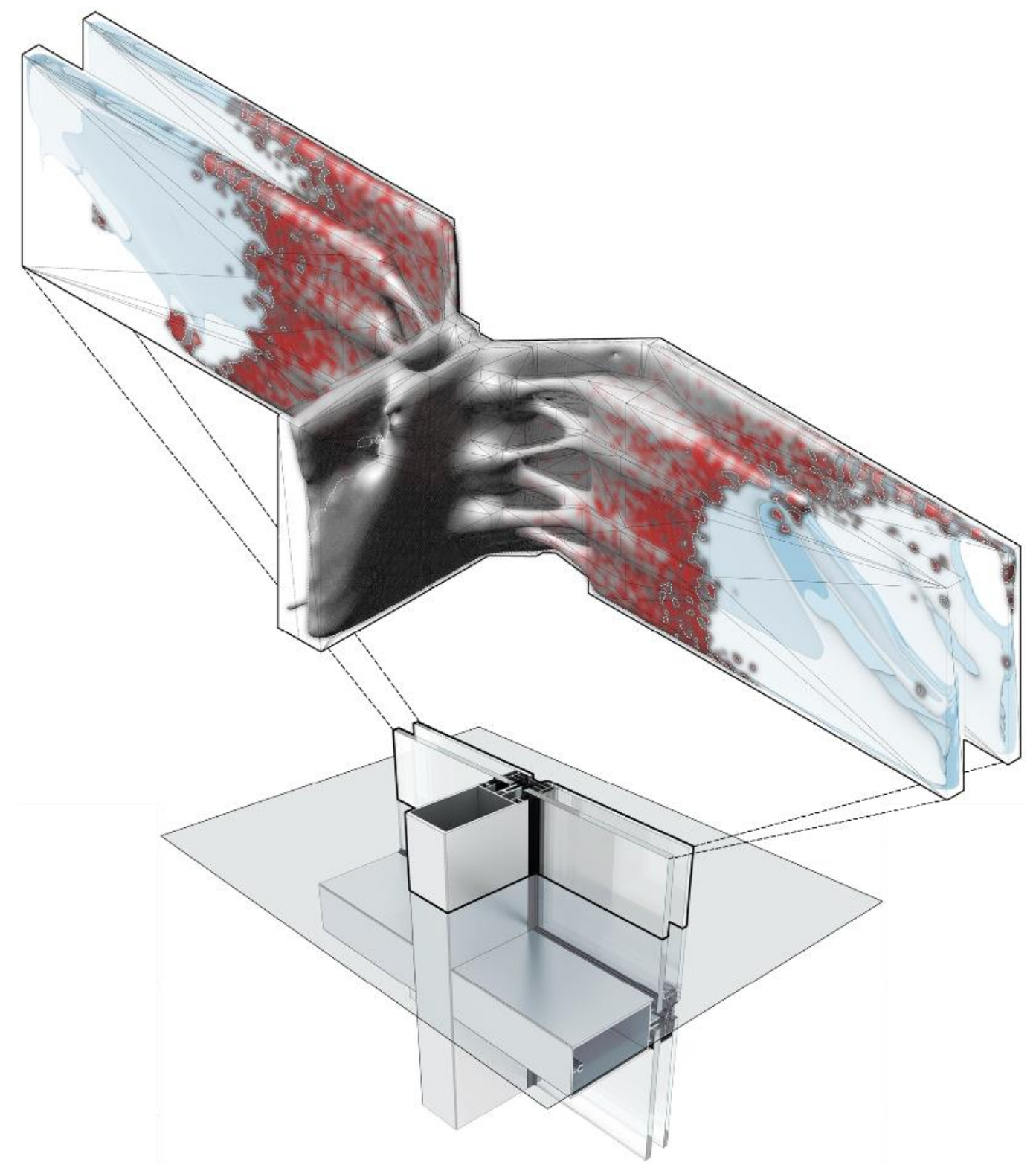

Figure 01 


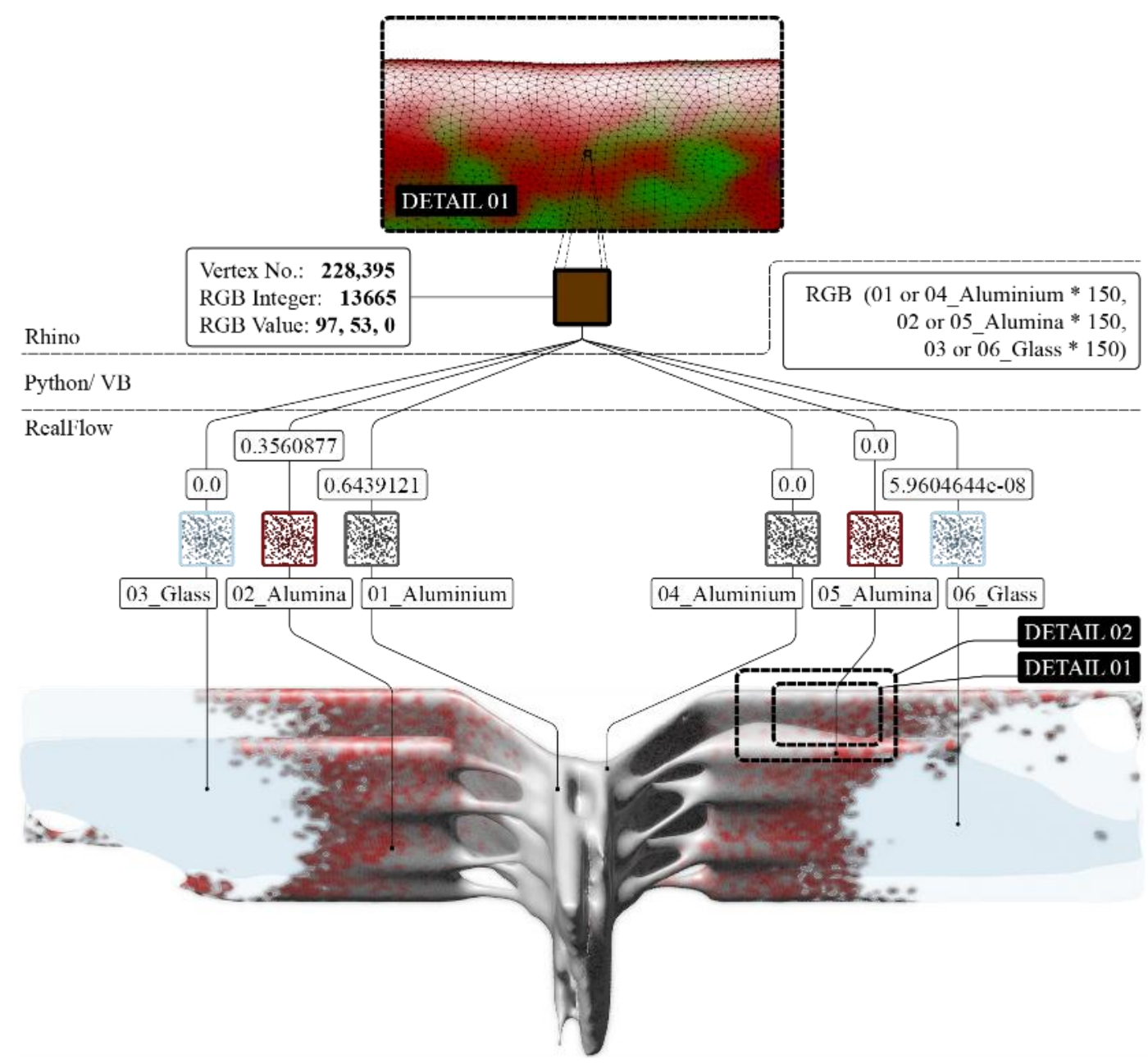

\section{Figure 02}

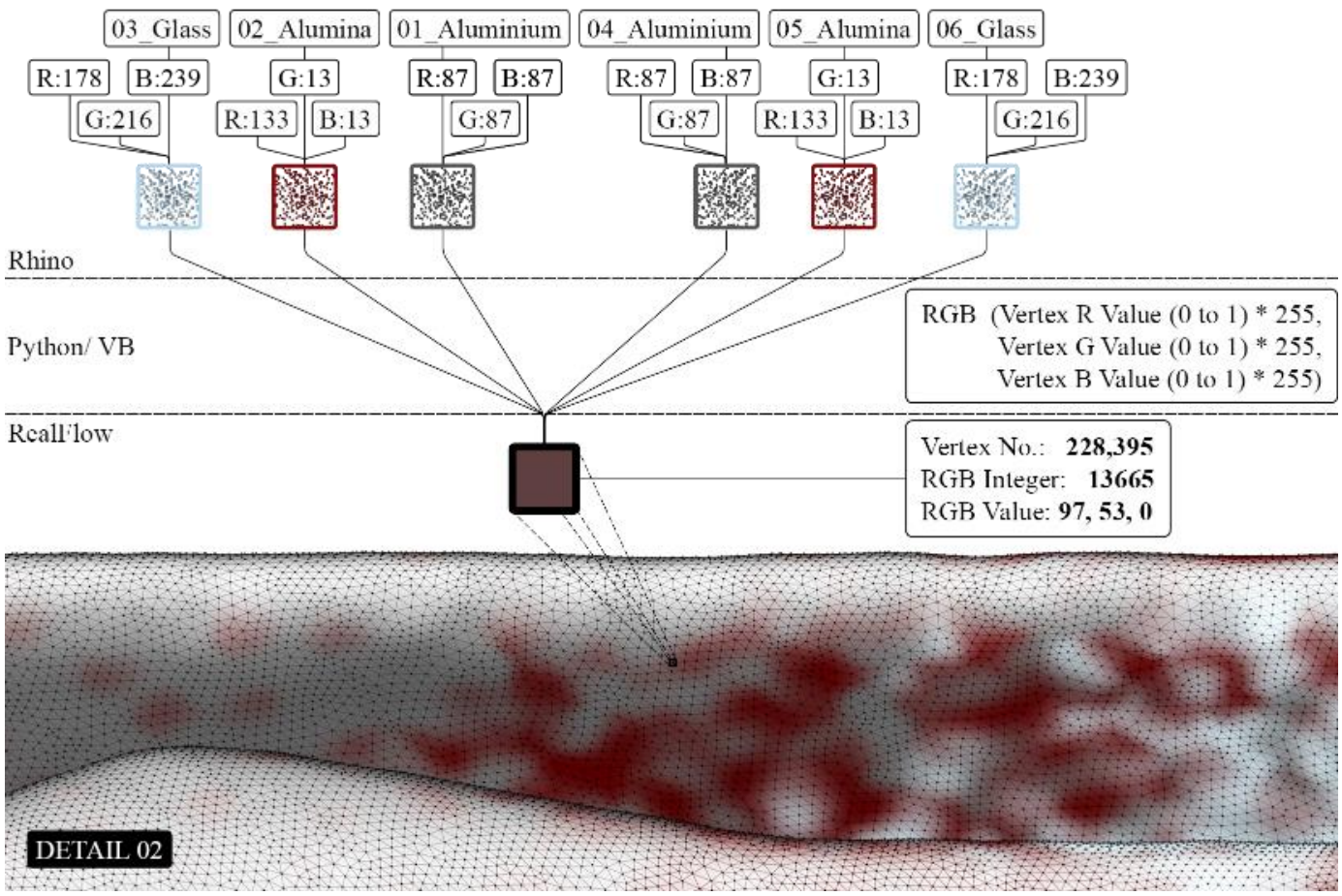

Figure 03 


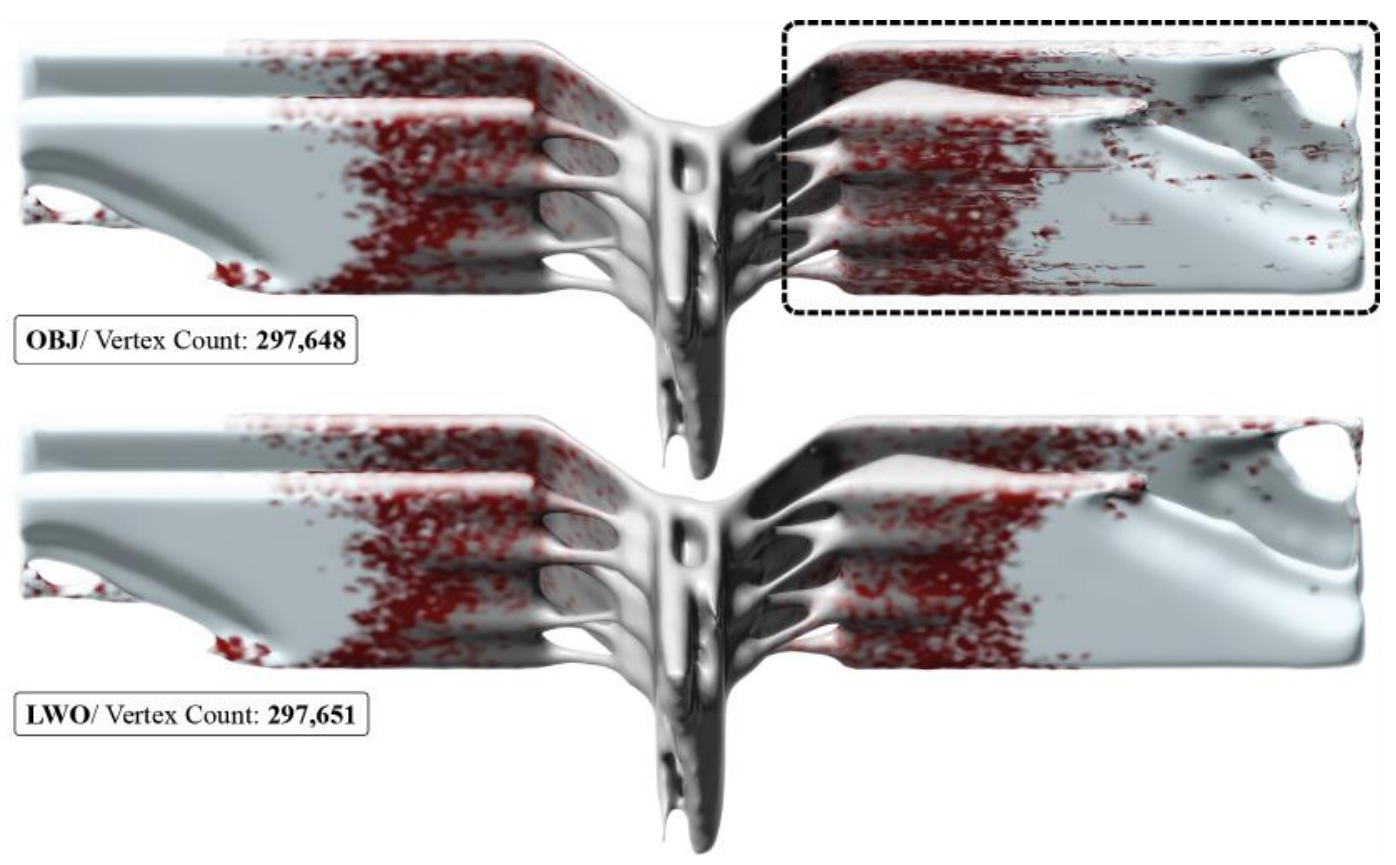

Figure 04

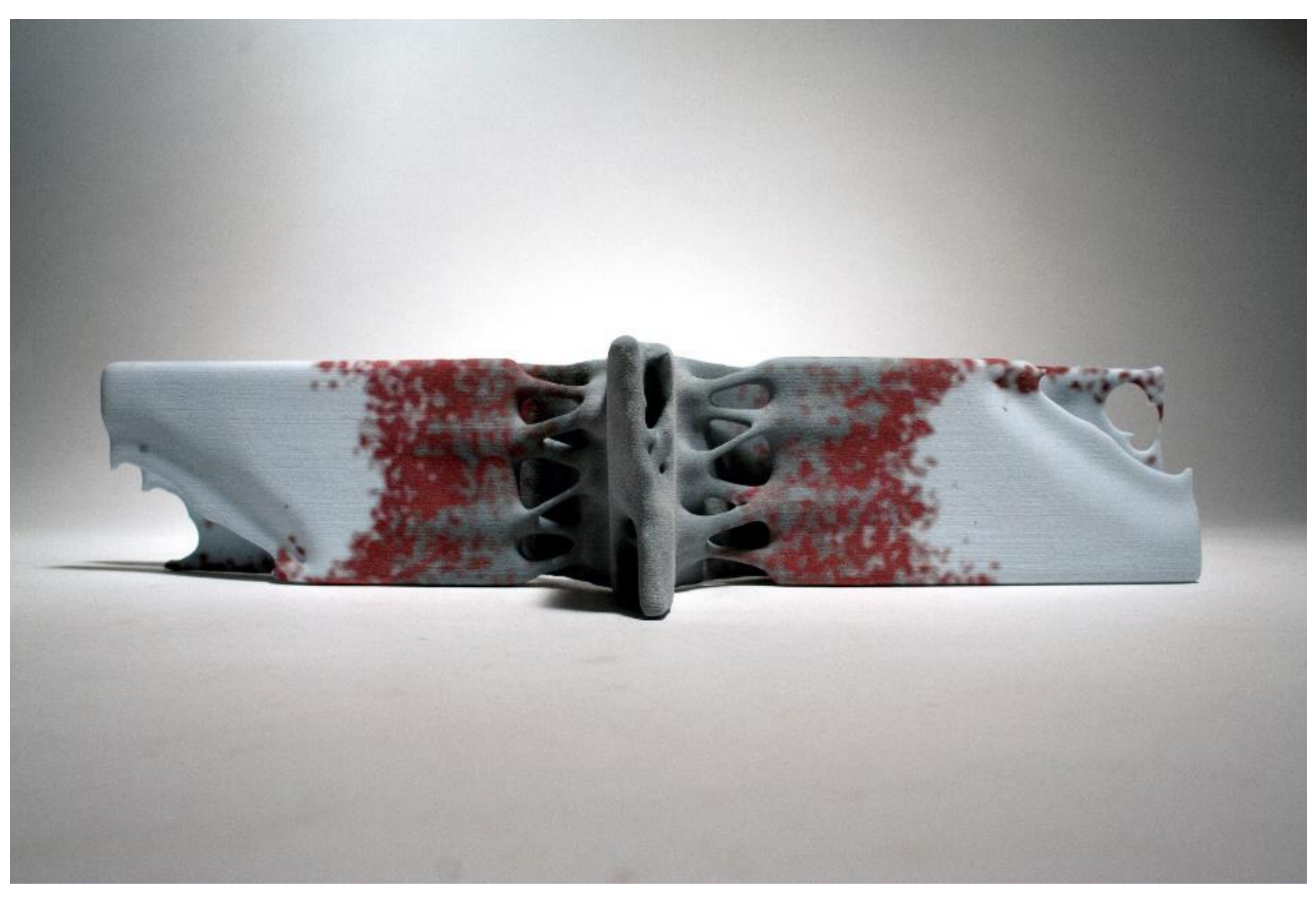

Figure 05 


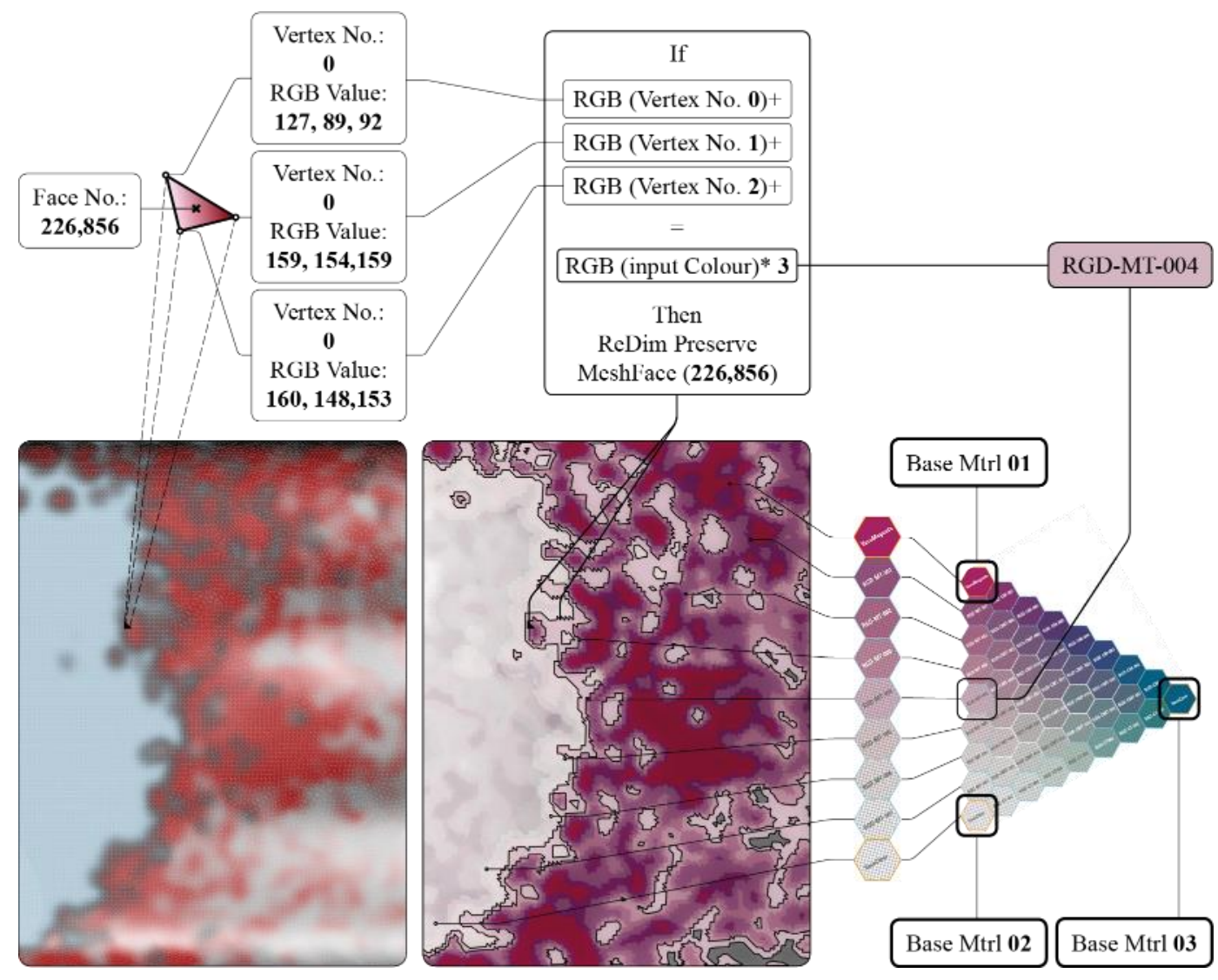

\section{Figure 06}

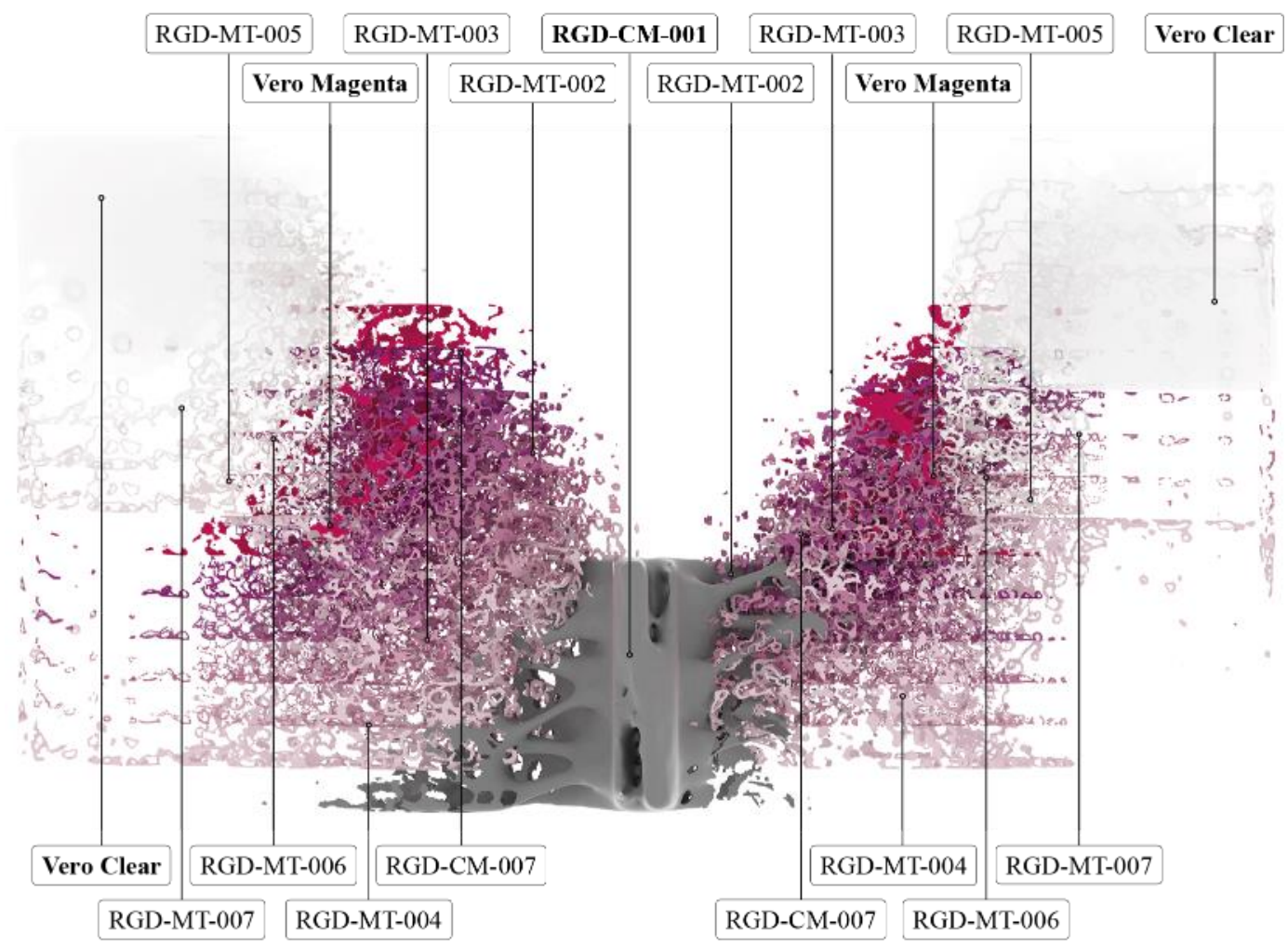

\section{Figure 07}



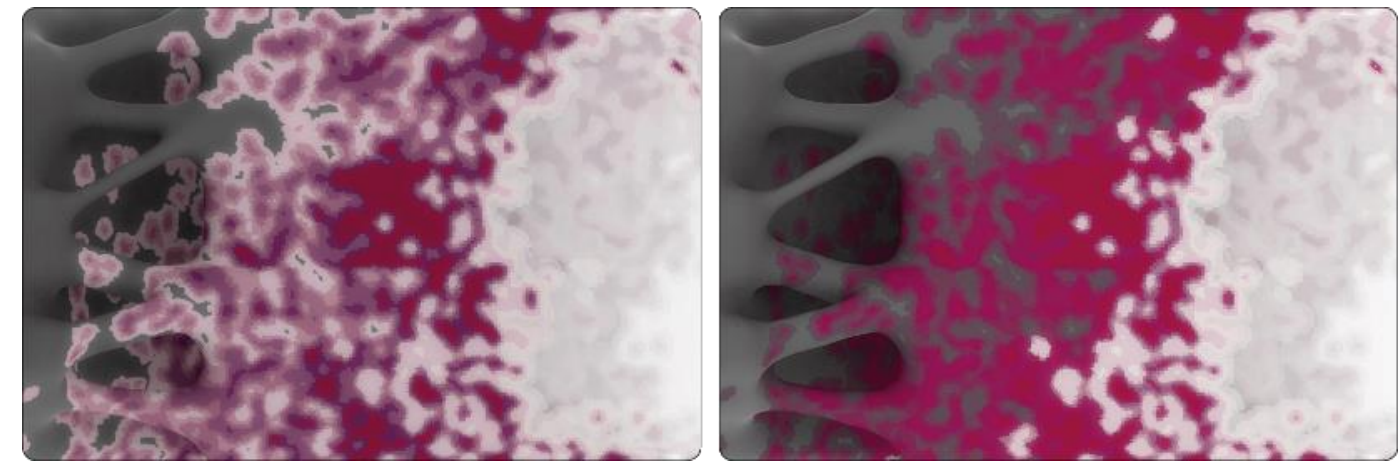

Figure 08

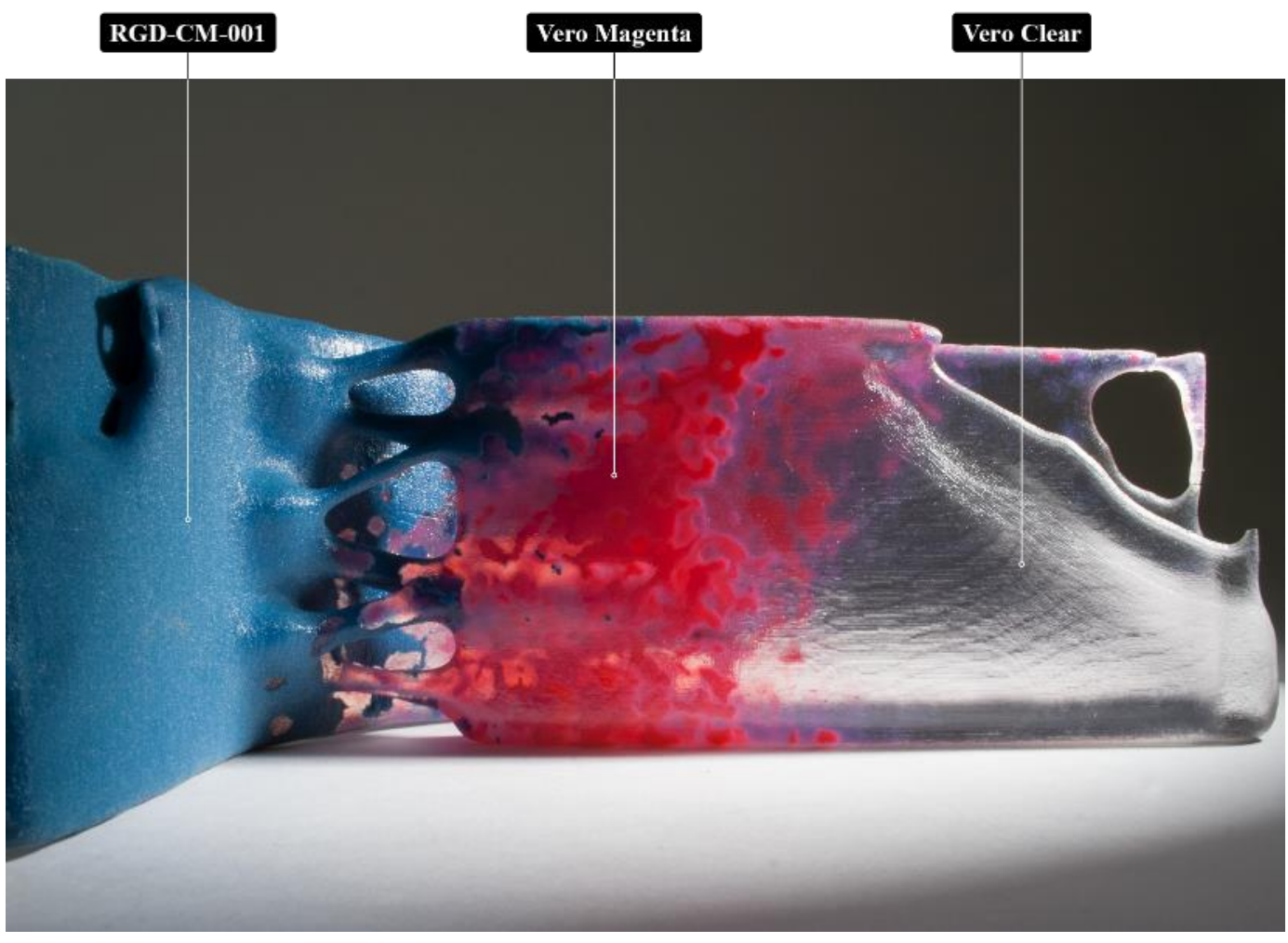

Figure 09 


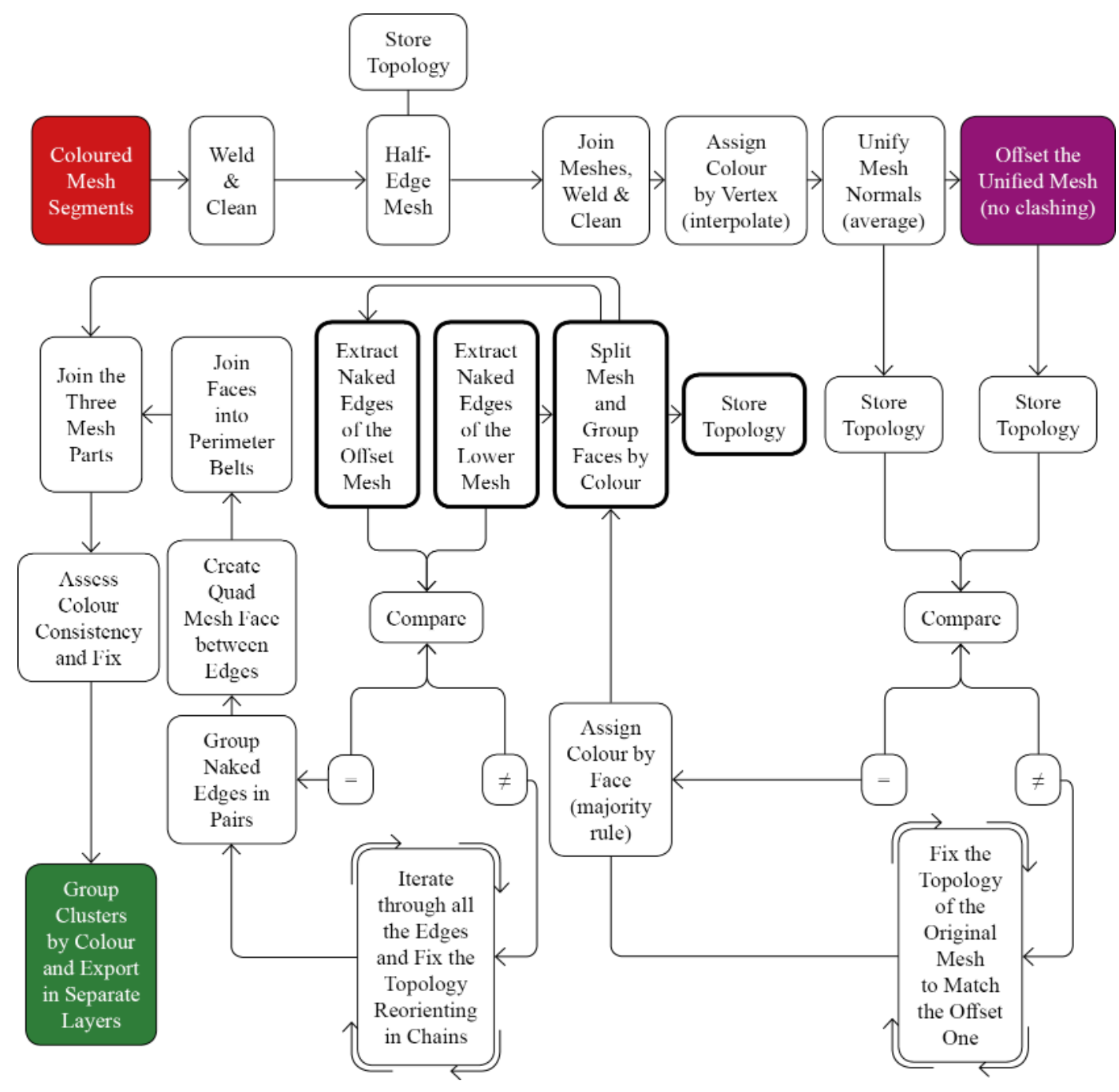

Figure 10 